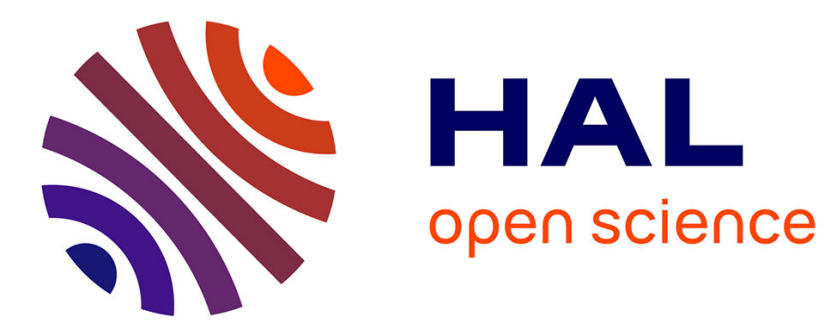

\title{
La labilité du réseau aux XVIIIe et XIXe siècles
}

Nicolas Verdier

\section{To cite this version:}

Nicolas Verdier. La labilité du réseau aux XVIIIe et XIXe siècles: Le cas de la Poste aux chevaux dans le quart nord-ouest de la France. Les Nouvelles de l'archéologie, 2009, 115, pp.13-17; 53-56. halshs-00372485

\section{HAL Id: halshs-00372485 https://shs.hal.science/halshs-00372485}

Submitted on 1 Apr 2009

HAL is a multi-disciplinary open access archive for the deposit and dissemination of scientific research documents, whether they are published or not. The documents may come from teaching and research institutions in France or abroad, or from public or private research centers.
L'archive ouverte pluridisciplinaire HAL, est destinée au dépôt et à la diffusion de documents scientifiques de niveau recherche, publiés ou non, émanant des établissements d'enseignement et de recherche français ou étrangers, des laboratoires publics ou privés. 


\title{
La labilité du réseau aux XVIIIe et XIXe siècles. \\ Le cas de la Poste aux Chevaux dans le quart nord-ouest de la France.
}

\author{
Nicolas Verdier CNRS \\ UMR 8504 Géographie-cités
}

\section{Introduction}

La Poste aux Chevaux est un service de transports rapides des personnes et de leur bagages qui se maintient durant environ quatre cents ans, soit entre la création des premiers relais au début du $\mathrm{XVI}^{\mathrm{e}}$ siècle, et la fermeture progressive des relais concurrencés par le chemin de fer à partir des années 1845. Elle n'est pas à confondre avec la Poste aux Lettres qui ne transporte que des objets, même s'il existe quelques relations contractuelles entre ces deux institutions. La Poste aux Chevaux, en tant qu'infrastructure territoriale se limite aux relais qui jalonnent les itinéraires. Même si elle utilise de plus en plus la route pavée ou empierrée construite au $\mathrm{XVIII}^{\mathrm{e}}$ siècle par les ingénieurs des Ponts et Chaussées, c'est bien l'ensemble des relais et non des tronçons qui est géré par cette institution; d'ailleurs entre les relais, les trajets peuvent être changeants, d'une période à une autre (Arbellot 1979). Mais l'impression qui domine encore aujourd'hui est celle d'une fixité des relais dans le temps long. Qui ne connaît un "relais de Poste", voire une auberge du "Cheval blanc" qui est décrite comme ayant été un relais de poste sans que la durée de cette fonction ne soit jamais interrogée. De même certaines villes sont décrites comme chef-lieu de généralité sous l'Ancien-Régime, comme si les deux institutions (la généralité et l'Ancien Régime) d'échelles et de natures différentes étaient corrélées. Pourtant, les changements dans la morphologie du réseau de la Poste aux chevaux, tout autant que dans l'écartement des distances entre les relais connaît entre le début du XVIIIe siècle et le milieu du XIXe siècle de mutations profondes. Dans les faits, moins de la moitié des relais se sont maintenus à l'identique. L'impression de longévité et de permanence doit donc laisser place à celle d'une labilité généralisée d'un réseau qui cependant se maintient, voire s'étend ${ }^{1}$. C'est à la localisation de cette labilité, qui produit une sorte de vibration du réseau que nous nous intéresserons ici.

\footnotetext{
${ }^{1}$ Par labilité, il faut ici entendre comme en chimie, l'idée de changement facile, plutôt que d'instabilité, concept qui renverrait plutôt à la thermodynamique.
} 


\section{Appréhender le réseau postal}

L'étude des réseaux de transport ou d'information au moyen d'un Système d'Information Géographique (SIG) est assez répandue en géographie mais reste restreinte en histoire. Il s'agit donc en même temps d'un travail très classique du point de vue de la géographie et d'un emprunt très particulier, du point de vue de l'histoire. La saisie dans le cadre du SIG de l'ensemble des données comprises dans les Livres de Poste qui paraissent régulièrement depuis 1708 s'est effectuée au travers du choix de coupes chronologiques, échelonnées tous les 25 ans depuis 1708, soit : 1708, 1733, 1758, 1783, 1810 (le Livre de Poste de 1808 est manquant) et 1833. Une saisie des lieux contenant un relais de poste, puis le tracé "à vol d'oiseau" entre deux relais successifs dans l'itinéraire postal ont été effectués. A cette première basse s'en est ajoutée une autre relative aux villes. Celle-ci, encore en cours de constitution, s'appuyant sur la base de Bairoch sur les villes européennes de plus de 5000 habitants (Bairoch, Batou, et Chèvre 1988), sur la base de Lepetit sur les villes françaises de plus de 10000 habitants (Lepetit, 1988), sur les recensements de 1793, 1800, 1806, et 1836², ainsi que sur la consultation de la bibliographie locale. Au totale, la base compte 267 localités qui comptent à un moment donné plus de 4000 habitants, dont pour l'instant 65 en 1700.

Le réseau postal se limite, dans la matérialité gérée par l'institution de la Poste aux Chevaux, aux relais, dont certains sont urbains (Verdier 2007). On connaît des cas extrêmes dans lesquels les créations de relais sont accompagnées de demandes faites aux Ponts et Chaussées d'aménager les routes existantes pour rendre possible le service. Ainsi, en 1771, Élie de Beaumont, qui sera l'avocat des Callas, obtient la création d'un relais de poste dans la paroisse de Canon, dont il est le seigneur. Il obtient que le tronçon nécessaire soit mis en état et que le chantier soir mis sous la responsabilité de l'ingénieur des Ponts et Chaussées, Maillet, de Falaise (Demangeon 1995, 66-71). Mais, ce n'est pas là le cas général. Le plus souvent on arrive péniblement à obtenir de l'ingénieur des Ponts et Chaussées, bon spécialiste des mesures terrestres, qu'il effectue le toisé de distances entre les relais. En dehors de cette question de mesure, l'ingénieur des Ponts et Chaussées peut être consulté par l'intendant pour le choix des routes. Ainsi, en 1761, l'intendant Le Bret demande son avis à l'ingénieur de Dol, Loiseleur sur l'ouverture d'un relais au Vivier. Celui-ci propose, sans être entendu, un autre trajet qui lui semble préférable (Gohier 1997, vol 1, 29). Mais le lien se limite à des mesures ou à des expertises, parfois demandées par une autorité administrative. Au-delà, rien ne dit que les Ponts et Chaussées entretiendront les routes s'ils ne les jugent pas

\footnotetext{
${ }^{2}$ Ces recensements sont consultables sur le site Cassini : $\underline{\text { http://cassini.ehess.fr/cassini/fr/html/index.htm }}$
} 
prioritaires, et donc que l'itinéraire se maintienne réellement tel qu'il a été créé. Les trajets effectivement pratiqués nous sont donc inconnus. Ne nous apparaissent que les modifications affectant les relais. Il reste néanmoins que le souci d'efficacité de l'institution de la Poste aux Chevaux la fait très certainement utiliser les meilleures routes à sa disposition.

\section{La distance, entre vol d'oiseau et sinuosités du terrain.}

Les résultats de l'enquête menée à l'aide du SIG nous donne accès à des informations qui si elles sont limitées n'en sont pas moins très éclairantes. En procédant par une saisie qui lie les relais à vol d'oiseau, on perd toute la rugosité des itinéraires, toutes les variations, tant menues que de grande ampleur qui éloignent de la ligne droite les trajets entre les relais. Notons sur ce point que cette question de différence entre pratique sur le terrain et trajet à vol d'oiseau n'a pas échappé aux acteurs de l'époque qui nous occupe ici. Le travail du corps des Ponts et Chaussées qui remplace les implantations routières passées décrites par leur sinuosité, par des routes droites et plates est l'exemple de cette préoccupation. Les planches de l'Atlas Trudaine (voir le texte de Stéphane Blond dans ce volume) montrent de ce point de vue la rectification notable des itinéraires durant le XVIIIe siècle. Le plus souvent l'ancienne routes sinueuse, apparaît non loin de la nouvelle, rectiligne. Inversement, les critiques adressées à la carte de Cassini la décrivent comme étant bonne à vol d'oiseau, mais n'ayant aucun sens lors des déplacements (Revel 1992 ; Pelletier 2002). Par ailleurs, l'ensemble des négociations opérées lors de la mise en place de nouveaux tarifs pour la Poste aux Lettres cette fois-ci, amène toujours un questionnement sur le rapport entre déplacement à vol d'oiseau et déplacement sur le terrain (Verdier 2002). Ainsi, le tarif postal de 1827, s'appuyant sur des réflexions du Bureau des longitudes, prétendra proposer un rapport constant entre ces deux types de distance. La question occupe donc les esprits de l'époque. Il n'en reste pas moins que la reconstruction de ces voies de communication du passé, dont on ne connaîtrait d'ailleurs pas l'usage réel nous est en très grande partie inaccessible. Toute reconstruction des routes de l'époque, comme celle effectuée par Théotiste Gohier, dans sa thèse sur la Poste aux chevaux dans la région malouine ne peut rien nous dire des pratiques réelles qui changent d'une année sur l'autre, en fonction de l'entretien, d'une saison à l'autre en raison des bourbiers que le mauvais temps amène, voire d'un conducteur à l'autre, en fonction de préférences dont on ne sait rien. C'est là la grande différence entre la route actuelle, qui s'impose aux usagers, et les routes du passé qui multiplient les possibilités tout en étant diversement sensibles aux aléas climatiques et à l'usure due au trafic. Pour la période médiévale, Céline Pérol parle ainsi de l'éffilochage des voies de communication (Pérol 2004), 
pour la période Moderne, Bernard Lepetit parlait du chevelu de ces mêmes voies (Lepetit 1984). Ce "monde que nous avons perdu", principalement au moment de la Restauration ${ }^{3}$ est celui de la Poste aux Chevaux. L'excellence de cette institution réside dans sa capacité à se maintenir malgré la fragilité des aspects matériels de son réseau. Dès lors, décrire dans leur morphologie routière fine les itinéraires serait comme une course vers l'horizon : un projet infini. La publication annuelle des Livres de Poste qui nécessitent de nombreuses retouches qui concernent non seulement les tableaux, mais encore les cartes, est déjà une tâche énorme pour l'éditeur des Postes (Jaillot). D'ailleurs comment décrire cette variabilité constante ? Et au-delà, pourquoi le faire ? ce serait comme dans le cas des circonscriptions d'Ancien Régime, insister sur la multiplicité, sur la diversité, en plaçant notre modèle de circonscription, ou de celui de réseau comme le meilleur, et celui du passé comme le moins bon. Il semble plus pertinent, tout d'abord de noter l'adéquation plus ou moins grande entre le modèle et le monde tel qu'il était, et ensuite de mesurer cette capacité à gérer un système éminemment complexe, ce qui ne retranche rien à sa fonctionnalité.

\section{Quelle croissance pour le réseau postal ?}

Dans le cadre défini par ces itinéraires à vol d'oiseau, nous pouvons affirmer que, au minimum, le réseau des routes de Poste aux Chevaux réparti sur le territoire français passe de 10000 à 27000 kilomètres entre 1708 et $1833^{4}$, et, si l'on se limite à un grand quart nordouest, que la longueur cumulée des distances à vol d'oiseau entre chaque relais du réseau passe de 3500 à 11000 kilomètres. La zone concernée qui correspond à $29 \%$ du territoire français métropolitain contient $40 \%$ de la longueur cumulée du réseau, tant en 1708 qu'en 1833. Il n'y a qu'entre 1783 et 1810 que cette proportion décroît de façon très faible, passant à $37 \%$ du total. La

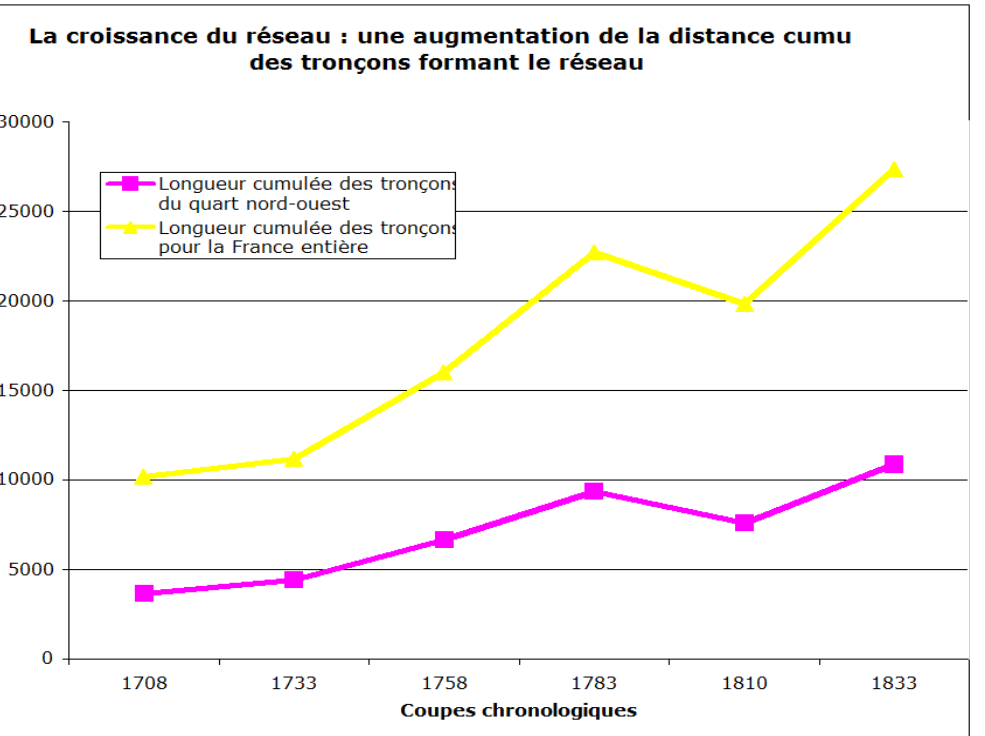

\footnotetext{
${ }^{3}$ La Restauration, puis la Monarchie de Juillet sont le moment d'une réduction drastique du nombre des voies au profit de quelques voies sur lesquelles la capacité d'entretien

${ }^{4}$ À titre de comparaison le réseau ferroviaire français atteint 25000 kilomètres vers 1875 . À cette date toutes les villes importantes du territoire sont desservies. En 1914, après le plan Freycinet de 1879 qui avait pour objectif de relancer la production industrielle, plus que d'équiper le territoire, on atteignait 36000 kilomètres.
} 
zone est donc très fortement équipée. Elle connaît par ailleurs une belle croissance puisque la distance cumulée triple presque entre 1708 et 1833 (multiplication par 2,75). On note une phase de rétraction au tournant des XVIIIe et XIXe siècles qui, pour dire les choses rapidement, est lié à la Révolution (désorganisation, crise économique, destruction des chaussées par le passage des troupes, redéploiement du service à l'extérieur du territoire...). Mais dès la Restauration, la croissance reprend. Cette croissance du réseau apparaît de façon évidente du côté des répartitions, à une densité faible en début de période (23 mètres de route par km2) on passe à une densité nettement plus forte en 1833 (700 mètres de route par km2). Cela ne veut évidemment pas dire grand chose, même dans cette zone densément occupée où des différences fortes existent. L'Isle de France, l'Artois et la Normandie connaissent des densifications considérables si on les compare à la Bretagne ou au Val de Loire. La croissance est donc hétérogène dans ses répartitions et l'on retrouve les zones les plus riches et les plus dynamiques qui arrivent en tête laissant derrière elle les régions plus faibles. Voilà le commentaire auquel on aboutit aisément à partir des données relatives aux distances cumulées et aux répartitions spatiales. Il serait possible d'affiner l'analyse en effectuant des zooms, ou en précisant des moments particuliers. Ainsi, il serait possible de développer le cas de la Bretagne pour laquelle malgré une densité qui reste assez faible, il existe une vraie croissance, puisque partant d'une centaine de kilomètres de tronçons, situés à ses limites en 1708 et 1733 , on en arrive dès 1758 à près de 1000 kilomètres, et finalement à 1800 kilomètres cumulés en 1833. En fait le conseiller d'État Orry, en 1738 ( $1^{\mathrm{er}}$ juillet$)$, soit l'année où il initie la corvée ${ }^{5}$ — ce qui permettra l'essor du réseau routier français — décide de faire profiter la Bretagne des avantages de la Poste aux Chevaux $^{6}$ (en grande partie contre la volonté locale) à partir de 1738. Il a l'année précédente effectué une série d'enquêtes en Bretagne de façon à être certain de la viabilité du projet et dès août 1738 il sera possible de joindre Rennes à Saint-Malo par la Poste (Gohier, 1997, 22-23) ${ }^{7}$. Mais cette approche par zoom reste dans la logique qui, soit pose de façon implicite que l'extension d'un réseau se fait par des ajouts successifs qui ne retranchent rien au passé, soit, en n'interrogeant pas la nature de la croissance, laisse penser qu'elle est un processus simple. Derrière cette absence d'interrogation se dissimule une conception d'un progrès qui fonctionnerait par simple accumulation. Les choses sont plus compliquées.

\footnotetext{
${ }^{5}$ La corvée est un impôt en nature qui oblige les imposables à travailler sur les routes, soit pour les construire, soit pour les remettre en état (13 juin 1738).

${ }^{6}$ AN F90 19004, arrêt du conseil du Roi, $1^{\text {er juillet } 1738 .}$

${ }^{7}$ AD Iles et Vilaine C 1980.
} 


\section{Décomposer la croissance pour appréhender le processus.}

En partant du constat de croissance d'une part et de celui de l'intense variation des pratiques entre relais, du fait de l'existence d'un chevelu de voies aux qualités variables, il convient de dépasser l'idée de croissance simple par ajouts successifs de tronçons pour poser à nouveaux frais la question de la nature de cette croissance. De ce point de vue, il est incontestable que la croissance du réseau passe d'abord par des ajouts successifs. L'augmentation du kilométrage cumulé des distances entre les relais nous en donne un aperçu. Mais inversement, cette croissance s'opère également par une série de suppressions de tronçons dans le réseau qui permettent d'avoir une conception beaucoup moins univoque du processus. En effet, la croissance du réseau s'opère par un retravail constant sur les itinéraires qui fait coexister les remplacements de tronçons existants avec la croissance par ajouts.

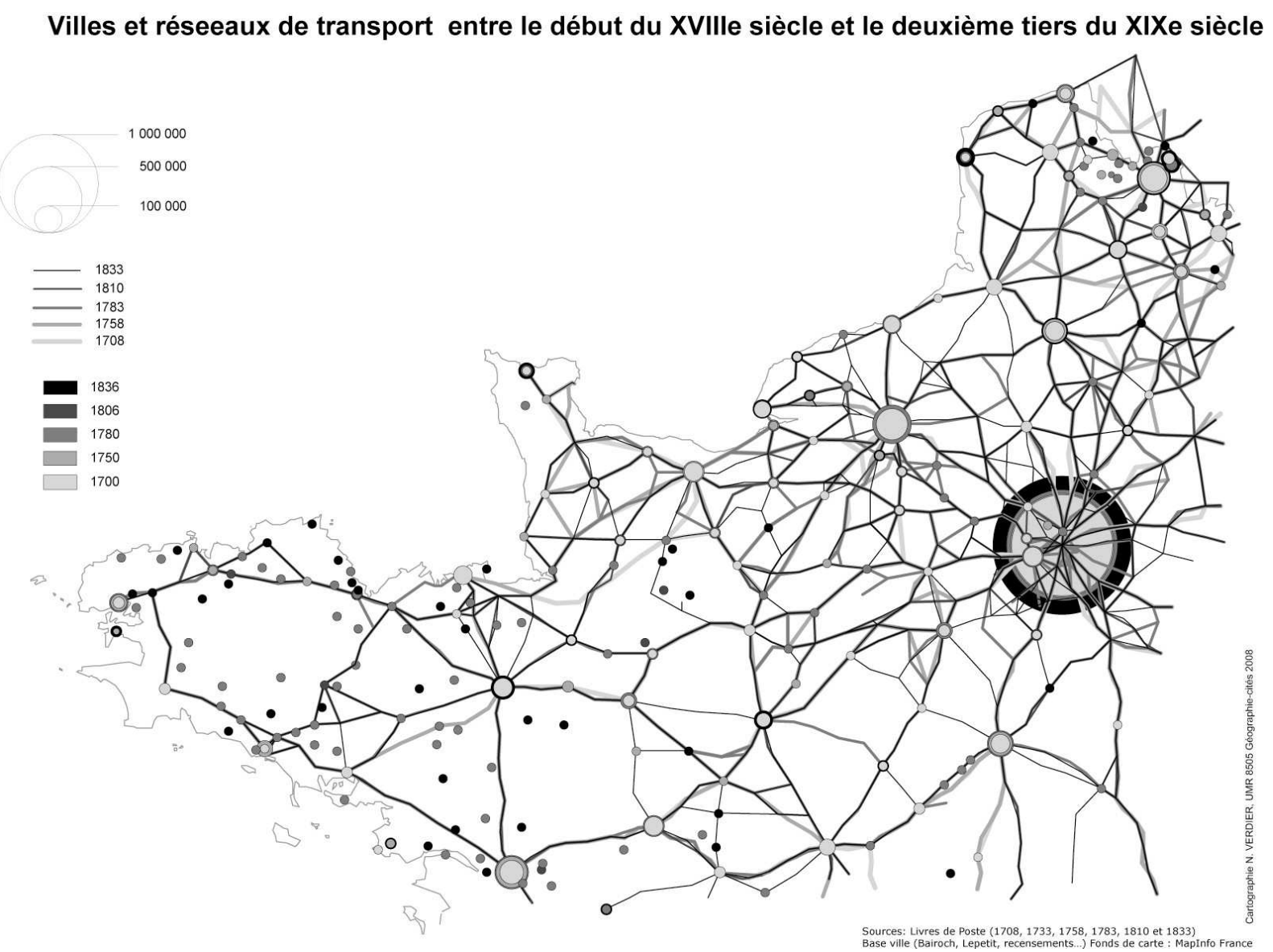

Avec Anne Bretagnolle, nous avons montré ailleurs (Bretagnolle et Verdier 2007), en comparant les tronçons ajoutés à ceux retranchés, et enfin à ceux qui se maintiennent que le jeu était particulièrement complexe : là où le réseau est le plus dense les changements (suppressions et ajouts) sont les plus nombreux, là où le réseau est le moins dense, les 
changements sont rares. En d'autres mots les réseaux peu denses sont stables, les réseaux denses sont labiles. Ce résultat, en soi intéressant, peut-être dépassé en s'intéressant à la répartition interne à ce mouvement général d'échelle au moins française. Le croisement entre la base réseau et la base ville sous-entend mon hypothèse : là où il y a des villes les points du réseau sont stables, en dehors, c'est l'instabilité. La carte qui cumule l'ensemble des réseaux et des localités de plus de 4000 habitants nous montre ces variations (Verdier 2008a). Les lieux de parcours sans fluctuation sont finalement rares, en dehors des tracés les plus récents, qui profitent à la fois de leur courte existence et d'un réseau routier en cours de sédimentation du fait de l'activité du corps des Ponts et Chaussées. Nous prendrons ici deux exemples de ces vibrations du réseau.

Le cas de la basse Normandie 1700-1830

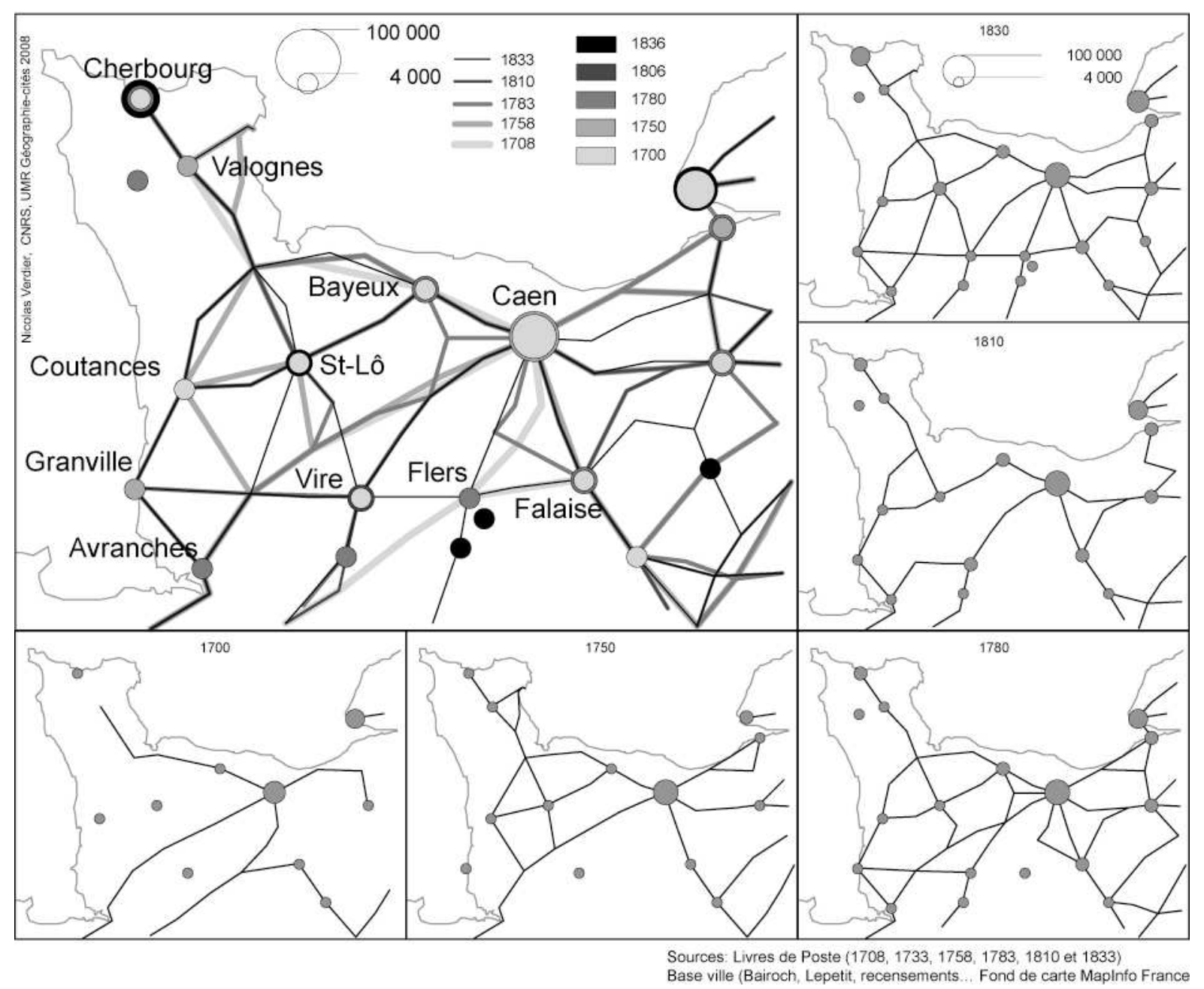

Le premier exemple est celui de la basse Normandie, le réseau, comme pour le reste de la France, se constitue progressivement en s'étoffant. Rien là de particulier, en revanche, 
l'intéressant réside dans les nombreuses fluctuations, cette sorte de vibration qui apparait dans la carte de synthèse, dans une zone délimitée par un groupe de ville qui contiendrait Caen, Bayeux, Saint-Lô, Coutances, Cherbourg, Valognes, et pour finir, même si je manque d'information sur la population de cette commune : Carentan, qui se trouve au carrefour entre Valognes et Saint-Lô et Bayeux et Coutances par le nord. Pour ce dernier lieu, on retrouve, en dehors de la proximité des localisations, une approche qui pourrait être complémentaire à celle d'Arnaud Chevallier. Celui-ci part des points pour reconstituer les réseaux, alors qu'ici le réseau permettrait peut-être de recomposer l'ensemble des points. En effet, la médiocrité des informations démographiques sur Carentan n'empêche pas ici de la penser comme étant à cette époque un carrefour important - il est vrai que ces deux critères ne sont pas nécessairement à lier de façon stricte. Mais, au-delà, ce qui importe ce sont les microfluctuations qui font que si la relation entre les villes se maintient, la route n'est jamais la même. Ainsi, l'axe Caen-Bayeux est modifié entre 1700 et 1750 , il est doublé entre 1750 et 1780, puis est à nouveau modifié entre 1780 et 1810. L'axe Bayeux-Carentan ne reste le même qu'entre 1750 et 1780 . Il va jusqu'à disparaître entre 1780 et 1810 , avant de réapparaître, sous une autre forme en 1830. L'axe Caen-Avranches n'est jamais le même tout au long de la période. Les mutations de détail sur cet axe sont une constante. Or ce tronçon participe à l'un des deux axes Paris-Rennes.

\section{L'exemple du Val de Loire 1700-1830}




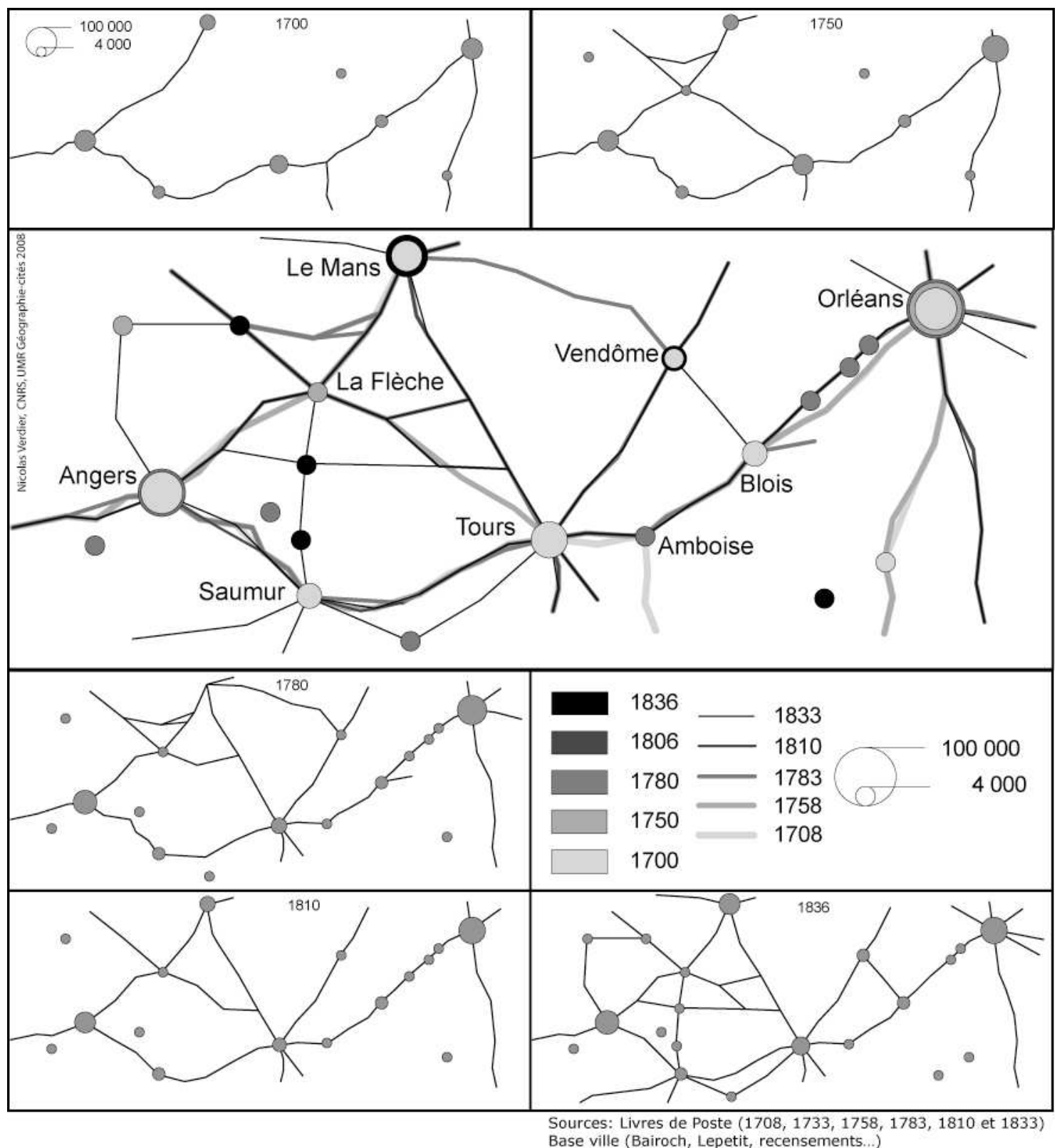

Le deuxième exemple est celui du Val de Loire, c'est-à-dire ici la région qui s'étend d'Orléans à Angers. Ce cas a été choisi préférentiellement à celui de la Seine car l'ensemble des méandres et les nombreux itinéraires alternatifs de l'axe entre Paris et la Manche, auraient rendu l'exemple peu lisible. Entre 1700 et 1750, les changements sont restreints. La modification principale concerne l'axe Saumur-Angers qui se trouve en partie déporté vers le Nord. Mais l'abandon du tronçon Amboise-Loches (qui s'explique peut-être par les destructions subies par les ponts d'Amboise en 1709 et 1710) ne suffit pas à redresser le trajet Blois-Tours, qui d'ailleurs continuera à passer par Amboise tout au long de la période étudiée. Ce point de passage sur la Loire, même s'il n'est pas exploité par la Poste aux Chevaux, attire donc jusqu'à lui l'itinéraire d'Orléans à Tours. L'un des processus les plus intéressants est le changement de rive qui s'effectue entre 1750 et 1780 dans le tronçon Orléans-Blois. L'axe 
général est maintenu, mais non seulement l'axe local change de rive, mais en même temps il semble capté par des localités qui connaissent une belle croissance dans le dernier tiers du XVIIIe siècle : Meung sur Loire, Beaugency et Mer. La série des aménagements que connaît le fleuve tout au long de la période explique probablement en partie ces divagations de routes, qui remplacent les divagations du fleuve contrôlé par les levées (Dion 1961). Au-delà, et cela même si le mouvement n'est pas aussi violent que dans le cas précédent, on notera que, à raison d'une coupe chronologique tous les vingt-cinq ans — même si ici celle de 1733 n'est pas présentée-, pas une seule fois le trajet d'Angers à Saumur n'est le même entre deux dates. L'ampleur des variations peut sembler restreinte, mais l'intensité, elle, est très forte. $A$ contrario, entre Blois et Amboise, l'axe est particulièrement stable dans le temps, ne connaissant qu'une fluctuation entre 1780 et 1810 .

\section{Conclusion}

Les grands axes de circulation glissent donc d'une époque à une autre, en fonction des besoins et des circonstances. Autrement dit, les axes routiers ont une temporalité. Les axes fluviaux et les littoraux jouent un rôle dans la localisation des voies, mais n'en donnent que les orientations générales. Nous l'avons vu, dans le cas de Bayeux-Carentan la ligne de l'intérieur des terres est progressivement abandonnée au profit d'une ligne plus côtière. De même, la tangente au Mont Saint-Michel dans l'axe Paris-Rennes se trouve progressivement abandonnée au profit d'un parcours passant par Vire et évitant Avranches.

Le deuxième élément de localisation routière semble être la ville. Celles-ci captent une partie des flux à leur profit. On notera ici que deux logiques se suivent. En début de période (17001750), les axes semblent en partie exister sans s'accrocher aux villes, du moins aux petites villes, ensuite, et cela malgré la multiplication des villes dans l'espace, rares sont celles à ne pas être desservies. Ces deux logiques correspondent peut-être à deux échelles. Jusqu'en 1750 la poste aux chevaux reste une émanation du pouvoir royal qui relie les grands centres à Paris. Après 1750, la logique commerciale, qui s'intéresse aux clientèles possibles, joue de plus en plus. L'intéressant réside ici dans les échelles de temps de la fluctuation. En 150 ans, les usages de la route se sont modifiés. On peut accepter l'hypothèse de la particularité de ce moment entre 1700 et 1830, juste avant la révolution ferroviaire. Il n'en reste pas moins que, à moyens relativement égaux - l'attelage n'évolue pas tant que cela, les croisements de chevaux n'amènent pas de nouvelles espèces si différentes des anciennes - la nature des usages routiers s'est trouvée remise en cause. Dès lors attribuer une fonction à une route sans 
en donner les temporalités est impossible. De même attribuer une fonction sans échelle n'est pas non plus possible. En effet si les grands axes se maintiennent, permettant des relations entre les grands centres - fonction relativement constante si l'on ne cherche pas à comprendre la nature de ces relations - en revanche à un échelon de compréhension locale, les fluctuations sont très probablement liées à des contextes différents, même si les temporalités des voies et des localités ne sont probablement jamais équivalentes. 


\section{Bibliographie du dossier Des Nouvelles de l'Archéologie « Du sentier à la route, une archéologie des réseaux viaires » coordonné par Sandrine Robert et Nicolas Verdier.}

Agache, R. 1978. La Somme pré-romaine et romaine. Amiens, Société des Antiquaires de Picardie, $515 \mathrm{p}$.

Allen, W. B., Liu, D., Singer, S. 1993. "Accessibility measures of US metropolitan areas », Transportation Research, 27B (6) : 439-449.

Les Annales, 1989., “Tentons l’expérience”, Annales E.S.C. nº. 1317-1323.

Antorne A. 2000. Le paysage de l'historien, Archéologie des bocages de l'Ouest de la France à l'époque moderne, Rennes, Presses universitaires de Rennes. 344 p.

Antorne, A., Vennel J.-P. 1994. « La voie royale et le paysage », Société archéologique et d'histoire de la Mayenne, fascicule 17. 35-57.

Arbellot G. 1973. « La grande mutation des routes de France au milieu du XVIII ${ }^{\mathrm{e}}$ siècle », Annales Économies Sociétés Civilisations, n³ : 765-791.

Arbellot, G. 1980. « Le réseau des routes de poste, objet des premières cartes thématiques de la France moderne », 104e congrès des sociétés savantes, Bordeaux 1979, Histoire moderne, Paris, Bibliothèque Nationale, $1: 97-115$.

Auray, J.-P., Bailly, A., Derycke, P.-H., Huriot, J.-M. 1994. Encyclopédie d'économie spatiale Concepts - Comportements - Organisations. Economica, Paris, 427 p.

Bairoch, P., Batou, J. et ChÈvre, P. 1988. La population des villes européennes de 800 à 1850, Genève, Droz.

Balard, M. dir., Laurioux, B. Le Jan, R., Le Mené, M. 2003. Dictionnaire de la France médiévale. Paris, Hachette, 287 p.

Bautier, R. H. 1987. « La route française et son évolution au cours du Moyen Age », Académie royale de Belgique, Bulletin de la classe des lettres et sciences morales et politiques, 1987, t. 73 : 70-104.

Benort, F. 1998. Modes d'occupation de l'espace protohistorique dans le Bassin moyen de l'Aisne, Mémoire de Maîtrise d'Archéologie, Université Panthéon-Sorbonne Paris I, 252 p.

Berge, C. 1973. Graphes. Éditions Gauthier-Villars, Paris, 400 p.

Berger, J. F., Bertoncello, F. , Braemer, F., Datvian, G., Gazenbeck, M. (dir.) 2005. Temps et Espaces de l'Homme en société, analyses et modèles spatiaux en archéologie. XXVe rencontres internationales 
d'archéologie et d'histoire d'Antibes, Antibes, Editions APDCA, 543 p.

Bertrand, A.1864. «Voies romaines en Gaule, voies des itinéraires. Résumé du travail de la Commission de la topographie des Gaules », Extrait de la Revue archéologique. Paris, Librairie académique, $61 \mathrm{p}$.

Bradley, R. 1991. "The pattern of change in British prehistory », in : T. Earle (ed.), Chiefdoms: power, economy and ideology. Cambridge : 44-70.

Bradley, R. et Hart, C. 1983. "Prehistoric Settlement in the Peak District during the Third and Second Millennia bc: A Preliminary Analysis in the Light of Recent Fieldwork », Proceedings of the Prehistoric Society, 49 : 177-193.

BRÉCHON, F. 2000. Réseau routier et organisation de l'espace en Vivarais et sur ses marges au MoyenAge. Thèse d'histoire. Lyon, Université de Lumière Lyon 2, 1368 p.

Bretagnolle, A. et Verdier, N. 2007. «L'extension du réseau des routes de poste en France de 1708 à 1833 », in Le Roux, M. (dir.), Postes d'Europe XVIIIe-XXIe siècles. Jalons d'une histoire comparée, Paris, Comité pour l'Histoire de la Poste, 2007, 155-171.

BRUAND, O. 2002. Voyageurs et marchandises aux temps carolingiens: les réseaux de communication entre Loire et Meuse aux VIIIe et IXe siècles. Bruxelles, De Boeck Université, 357 p.

Bruinsma, F., Rietveld, P. 1993. "Urban agglomerations in European infrastructure networks », Urban Studies, 30 : 919-934

Brun, P., Guichard, Y. et Le Goff, I. 2005. « Les tombes à incinération de l'âge du Bronze et du Ier âge du Fer dans le bassin de l'Aisne : Observations préliminaires. ", in : Les pratiques funéraires à l'âge du Bronze en France, Actes de la table ronde de Sens-en-Bourgogne, 10-12 juin 1998. Société Archéologique de Sens, Comité des travaux historiques et scientifiques : 477-492.

Burgess, C. 1980. The Age of Stonehenge. Londres, J.M. Dent \& Sons Ltd, 402p.

CAPOT-Rey, R. 1946. Géographie de la circulation sur les continents, Paris, Gallimard, 296 p.

Castex, J, Céleste P. et Panerai Ph. 1979. Lecture d'une ville : Versailles. Paris, Editions du Moniteur, col. Architecture "Etudes", 235 p.

Cattan, N., Grasland, C. 1997. Les différentiels d'accessibilité des villes moyennes en France. Rapport de recherche pour le METL, CNRS, Laboratoire ParisGeo, 115p.

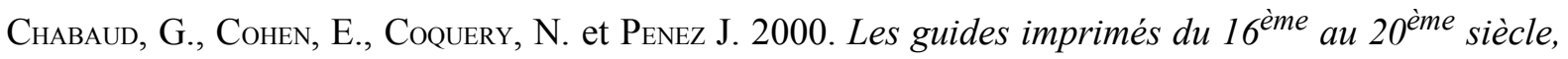
villes, paysages voyages, Paris, Belin, $704 \mathrm{p}$.

Chapelon, L. 1997. Offre de transport et aménagement du territoire - Evaluation spatio-temporelle des projets de modification de l'offre par modélisation multi-échelles des systèmes de transport. Thèse 
de doctorat en Aménagement, Université François Rabelais de Tours, 558 p.

Chapelot, J. 1993. "L'habitat rural : organisation et nature », in L'Ile-de-France, de Clovis à Hugues Capet, du Ve au Xe siècle, catalogue de l'exposition, Guiry-en-Vexin, Musée archéologique, 178-199. Снаротат, G. 1981. «La voie protohistorique sud de la croisée de Vienne. Essai de reconstitution de son tracé jusqu'à Marseille ». Revue Archéologique de l’Est, 1981, Tome XXXII, n¹25-126 : 83-91

Chartrain, A. 2007. «Parcellaires fossiles et faits funéraires dans l'espace et le temps sur le territoire rural de Montpellier » Medieval Europe Paris 2007, http://www.archeogeographie.org

Chevalier, A. 2005. Les enclos funéraires protohistoriques de Basse-Normandie : Essai d'analyse spatiale, Mémoire de Maîtrise d'Archéologie, Université Panthéon-Sorbonne, Paris I. 175 p.

Chevalier, A. 2006. Recherche d'une structuration de l'espace protohistorique en Basse-Normandie : exercice d'analyse spatiale, Mémoire de Master II Recherche, Université Panthéon-Sorbonne, Paris I. $125 \mathrm{p}$.

Chevallier, R. 1972. Les voies romaines. Paris, Armand Colin, 313 p.

Chevallier, R. 2000. Lecture du temps dans l'espace. Topologie archéologique et historique Paris, Picard, 229 p.

Chevallier, R. dir. 1983. Les voies anciennes en Gaule et dans le monde romain occidental, Colloque tenu à Paris, ENS, 5-6 juin 1982 ». Caesarodunum nXVIII, Tours, Université de Tours, 487 p.

Chouquer, G. (dir.) 1996. Les Formes du paysage, Tome I : études sur les parcellaires : pré-actes du colloque d'Orléans (mars 1996). Paris, éditions Errance, 222 p.

Chouquer, G. (dir.) 1996. Les Formes du paysage, Tome.2: Archéologie des parcellaires, Paris, Editions Errance, 263 p.

Chouquer, G. (dir.) 1997. Les Formes du paysage, Tome 3 : L'analyse des systèmes spatiaux, Paris, Editions Errance, 198 p.

Chouquer, G. 1996. «Parcellaires et longue durée; Points de repères historiques et problèmes d'interprétation », in: Chouquer G. dir. Les Formes du paysage, Tome 2 : Archéologie des parcellaires. Actes du colloque d'Orléans (mars 1996). Paris, Editions Errance, 1996 : 201-223.

Chouquer, G. 2000. L'étude des paysages. Essais sur leurs formes et leur histoire. Editions Errance. Paris. 208 p.

Chouquer, G. 2006. «L'émergence de la planimétrie agraire à l'Age du Fer », in : Chouquer G. dir. Etudes Rurales $\mathrm{n}^{\circ} 175-176: 29-52$.

Chouquer, G. et al. 1996. « La morphologie agraire et les paysages de la plaine des Tilles et de l'Ouche (Côte-d'Or) ». Chouquer, G. (dir.) Les Formes du paysage, Tome I : études sur les parcellaires : pré- 
actes du colloque d'Orléans (mars 1996). Paris, éditions Errance,: 32-49.

Coquery, N. L'Hôtel Aristocratique. Le marché du luxe à Paris au XVIIIe siècle, Paris, Publications de la Sorbonne. $444 \mathrm{p}$.

Dainville F. (de) 1964. Le langage des géographes, termes signes, couleurs des cartes anciennes, 1500-1800, Paris, Picard. XX-384 p.

Daremberg, Ch. et Saglio, E. 1969. Dictionnaire des antiquités grecques et romaines : d'après les

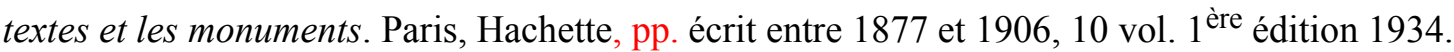

Daveau, I. et Yvinec, J. H. 2002. «L'occupation gallo-romaine du site de Fontenay-en-Parisis "La Lampe" (Val-d'Oise) : établissement agricole spécialisé ou lieu de culte ? » in : Revue Archéologique du Centre, Tome 41, Tours, $2002: 129-172$.

Decoupigny, C. 2003. "Couloirs de trafics et émissions de polluants », in: Mathis, Ph. (dir.) 2003. Graphes et réseaux, modélisation multiniveau. Paris, Lavoisier: 187-211.

Demangeon, P. 1995. La Poste en Basse Normandie, Vire, Charles Corlet éditions. 439 p.

DenFord, G.T. 1975. « Economy and location of Bronze Age "arable" settlements on Dartmoor », Bulletin of the Institute of Archaeology, 12 : 175-196.

DervilLe, A. 1978. «La première révolution des transports continentaux, (c.1000 - c. 1300.) », in : Annales de Bretagne et des Pays de l'Ouest 85 (1978) : 181-205

Desbordes, J. M. et Barrière, B. 1983. "Anciens itinéraires entre Limousin et Périgord », in : Chevallier, R. dir. 1983. "Les voies anciennes en Gaule et dans le monde romain occidental, Colloque tenu à Paris, ENS, 5-6 juin $1982 »$. Caesarodunum $n^{\circ} X V I I I$, Tours, Université de Tours, : 189-199.

Desloges, J. à paraître. Histoire du peuplement de la Basse-Normandie, L'apport de la prospection aérienne 1988 - 2004. Direction Régionale des Affaires Culturelles de Basse-Normandie, Service Régional de l'Archéologie.

Devroey, J. P. 1984. «Un monastère dans l'économie d'échanges : les services de transport à l'abbaye Saint-Germain-des-Prés au IXe siècle », in : Annales Economie Sociétés Civilisation, 39, 1984 : 570589

Dion, R. 1961. Histoire des levées de la Loire, Paris, 312 p.

DindJian, F. 1991. Méthodes pour l'archéologie. Paris, Armand Colin, 401 p.

Dunin-Wasowicz, T. 1982. «Les routes médiévales en Europe centrale : bilan et perspectives », in :

Durand-Dastès, F. et al. 1998. Archaeomedes. Des oppida aux métropoles. Archéologues et géographes en vallée du Rhône. Paris, Anthropos, Economica, 280 p. 
EJstrud, B. 2005. «Cost surface analysis and ancient roads : a comparison », in : Berger, J. F., Bertoncello, F. , Braemer, F., Datvian, G., Gazenbeck, M. (dir.) 2005. Temps et Espaces de l'Homme en société, analyses et modèles spatiaux en archéologie. XXVe rencontres internationales d'archéologie et d'histoire d'Antibes, Antibes, Editions APDCA : 135-139.

FAVIER, J. 1993. Dictionnaire de la France médiévale. Paris, Fayard, 982 p.

Favory, F. , Fiches, J.L., Raynaud, C. et Clatot, M. « La dynamique de l'habitat gallo-romain dans la basse vallée du Rhône », in DuRAnd-DASTĖs, F.et al. 1998. Archaeomedes. Des oppida aux métropoles. Archéologues et géographes en vallée du Rhône. Paris, Anthropos, Economica : 73-115.

Favory, F., Parodi, A., Poupet, P. et Raynaud, C. 1994. «Lunel-Viel et son territoire », in : Favory F. et Fiches J. L. (dir.). Les campagnes de la France méditerranéenne dans l'Antiquité et le haut MoyenAge, DAF n², Paris, Editions de la maison des sciences de l’Homme, 1994 : 163 - 244

Febvre, L. 1922. La terre et l'évolution humaine, introduction géographique à l'histoire, Paris, Renaissance du livre, $470 \mathrm{p}$.

FourQuin, G. 1964. Les campagnes de la région parisienne à la fin du Moyen-Âge, Paris, PUF, 590 p.

FreEman, L. 1979. « Centrality in social networks - Conceptual clarification ». Social Networks, $\mathrm{n}^{\circ} 1$ : 215-239

Garmy, P., Kaddouri, L., Rozenblat C. et Schneider, L. 2005 « Logiques spatiales et « systèmes de villes » en Lodévois de l'Antiquité à la période moderne », in BERGER, et al. (dir.). Temps et Espaces de l'Homme en société, analyses et modèles spatiaux en archéologie. XXVe rencontres internationales d'archéologie et d'histoire d'Antibes, Antibes, Editions APDCA : 225-236.

GleYze, J.-F. 2005. La vulnérabilité structurelle des réseaux de transport. Thèse de doctorat de Géographie - Université de Paris VII Denis Diderot, 848 p.

Gleyze, J.-F. 2007a. « Effets spatiaux et effets réseaux dans l'évaluation d'indicateurs sur les nœuds d'un réseau d'infrastructures ». CyberGeo, n³70, 24 p. http://www.cybergeo.eu/

GleYZE, J.-F. 2007b. « Reducing functional network vulnerability - Structural indicators to anticipate the structural reorganization of a damaged transportation network », in : Actes de la 15ème édition de la Conférence Européenne de Géographie Théorique et Quantitative - ECTQG, Montreux, Suisse, 4 p.

GoHIER, Th. 1997. La poste aux chevaux dans la région malouine (1738-1870), Thèse de doctorat de Sciences Sociales de l'universtié de Haute-Bretagne Rennes II, sous la direction de Claude Nières, 1997, 2 vol.

Gould, S.J. 2005. Le renard et le hérisson, Comment combler le fossé entre la science et les humanités. Trad. N. Witkowski, Paris, Seuil, 364 p. 
Grenier, A. 1985. Manuel d'archéologie gallo-romaine. Tome 2, L'archéologie du sol. Navigation-

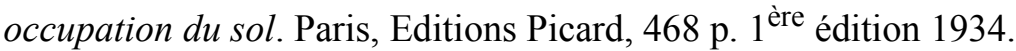

Groupe Dupont, 2004, Géopoint 2004, La forme en géographie, Avignon, Groupe Dupont, 505 p.

Guilaine, J. 1991. « Vers une préhistoire agraire » in : J. Guilaine (dir.), Pour une archéologie agraire, Paris, Armand Colin : 31-80.

Guilaine, J. 2000. « Changeons d'échelles : pour la très longue durée, pour de larges espaces », Etudes rurales, 153-154:9-21.

Guillaumet, J. P. 1996. L'artisanat chez les gaulois. Paris, Errance, 127 p.

Guillerme, A., 1984, Corps à corps sur la route. Les routes, les chemins et l'organisation des services $d u$ XIXe siècle, Paris, Presses de l'ENPC, 172 p.

Herce Vallejo, M. 1983. "La utilización de indicadores topológicos en el analisis de redes de communicaciones - Ensayo sobre la red de carreteras de Cataluña ». Documents d'Anàlisi Geogràfica, $n^{\circ} 3: 3-46$

Herrmann, P. 2007. Itinéraires des voies romaines. Paris, Editions Errance, 275 p.

Hodson, F.R. 1971. «Cluster analysis Numerical typology and prehistoric archaeology », in : F.R. Hodson, D.G. Kendall et Tautu P. (éds), Mathematics in the Archaeological and Historical Sciences. Proceedings of the Anglo-Romanian Conference, Mamaia 1970, organized by The Royal Society of London, and The Academy of the Socialist Republic of Romania, Edinburgh, Edinburgh University Press : $30-45$

HuRE, A. 1978. Le Sénonais gallo-romain, Bruxelles, Editions Culture et civilisation, 422 p.

Huriot, J.-M., Perreur, J. 1994. « La centralité / L'accessibilité », in : (Auray J.-P. et al. 1994) : 4758.

INGRAM, D.R. 1971. "The concept of accessibility : a search for an operational form ». Regional Studies, $5: 101-107$

JEANNET, N. (à paraître). « Contributions pratiques d'une géostatistique raisonnée en environnement : méthodes et application à la cartographie régionale de la pollution par le dioxyde d'azote $\left(\mathrm{NO}^{2}\right)$ en Alsace », in : Aubry, L. et Soulier, P. (dir.): Les approches spatiales des méthodes statistiques appliquées à l'archéologie. Journée d'étude organisée par le 13 décembre 2007 à la MAE de Nanterre. Cahiers transversaux de l'UMR 7041

Johnson, A.W. et Earle, T. 1997, 2000 (2e éd). The Evolution of Human Societies. From Foraging Group to Agrarian State. Stanford, Stanford University Press, 456 p.

JoLy O. 1999. Geographical position : State of french art of spatial accessibility indicators. SPESD - 
France, Groupe de travail I. 1, 23 p.

Journées intenationales d'histoire. Centre culturel de l'abbaye de Flaran, 1982. «L'homme et la route en Europe occidentale au Moyen Age et aux Temps Modernes », in : Cahier de Flaran, $n^{\circ} 2$, Auch. 303 p.

Jullian, C. 1920. Histoire de la Gaule, tome V, Paris Hachette, 381 p.

Jung, C. 1999. Morphogénèse, fonctions et évolution de la centuriation B d'Orange et essai de restitution diachronique des paléopaysages du Tricastin (Drôme-Vaucluse). Thèse de doctorat, Université de Tours, $366 \mathrm{p}$.

Konvitz J.F. 1987. Cartography in France, 1660-1848, Science, Engineering and Statecraft, Chicago/ London, University of Chicago press. 194 p.

Laffont, P. Y et Bréchon, F. 2008. «Châteaux, incastellamento et réseau viaire au Moyen-Age en France méridionale », in : Conpatangelo-Soussignan R., Bertrand J. R., Chapman J. et Laffont P. Y. (dir.). Marqueurs des paysages et systèmes socio-économiques. Actes du colloque COST du Mans (7-9 décembre 2006), Rennes, Presses Universitaires, 2008 : 149-163.

Lамвот, B. 1996. « Les Rèmes à la veille de la romanisation. Le porcien au Ier siècle avant J. C. », in : Bayard D. et Collard J. L. (dir.), Revue Archéologique de Picardie, $n^{\circ}$ spécial, 11/1996, Amiens : 1338.

Lavigne, C. 1997. «Parcellaires de fondation et parcellaires de formation à l'époque médiévale en Gascogne. Clefs de lecture et problèmes d'interprétation », in : ChOuQuer G. (dir.) 1997 Les Formes du paysage, Tome 3 : L'analyse des systèmes spatiaux, Paris, Editions Errance : 149-159.

LebecQ, S. 1989. « La Neustrie et la mer », in : H. Atsma (publ.), La Neustrie, Les pays au nord de la Loire de 650 à 850. Jan Thorbecke Verlag Sigmaringen, 1989 : 406-440.

Legros, R. 1983. «Les chemins des Rèmes », in : Chevallier R. (dir.) 1983 Les voies anciennes en Gaule et dans le monde romain occidental, Colloque tenu à Paris, ENS, 5-6 juin 1982 ». Caesarodunum $n^{\circ}$ XVIII, Tours, Université de Tours : 269-289.

Leone, F., Aste, J.-P., Velasquez, E. 1995. «Contribution des constats d'endommagement au développement d'une méthodologie d'évaluation de la vulnérabilité appliquée aux phénomènes de mouvements de terrain ». Bulletin de l'Association de Géographes Français - "Croissance urbaine et risques naturels », Vol. 4, 72ème année : 350-371.

LEPETIT, B. 1984. Chemins de terre \& voies d'eau, réseaux de transport organisation de l'espace, Éds. de l'E.H.E.S.S., Paris. 148 p.

Lepetit, B. 1988. Les villes dans la France moderne (1740-1840), Paris, Albin Michel. 490 p.

LeturcQ, S. 1997. «La route et le paysage. Dynamique et stabilité des réseaux routiers beaucerons 
entre Etampes et la forêt d'Orléans », in : Chouquer G. (dir.) 1997 Les Formes du paysage, Tome 3 : L'analyse des systèmes spatiaux, Paris, Editions Errance: 66-78.

LeURENT, F. 2006. Structures de réseau et modèles de cheminement. Lavoisier, 394 p.

Levy, J. et Lussault, M. (dir.), 2003. Dictionnaire de la géographie et de l'espace des sociétés. Paris, Belin, 1033 p.

Livet, G. 2003. Histoire des routes et des transports en Europe. Des chemins de Saint-Jacques à l'âge d'or des diligences. Strasbourg, Presses universitaires, 608 p.

LLobera, M. 2003. " Estending GIS-based visual analysis: the concept of visualscapes 》. International Journal of Geographical Information Science Vol. 17/1 : 25- 48.

Lot, F. et FawtieR, R. 1932. Le premier budget de la monarchie française, Paris, 259 p.

Loubès, G., « Routes de la Gascogne médiévale », Journées intenationales D’histoire. Centre culturel de L'ABbaye de Flaran, 1982. «L'homme et la route en Europe occidentale au Moyen Age et aux Temps Modernes », in : Cahier de Flaran, $n^{\circ} 2$, Auch : 33-56.

Luchaire, A. 1885. Les actes de Louis VII, Paris, Editions Alphonse Picard, 527 p.

Lundmark, H. 1984. « The identification of tribal hierarchies », in : K. Kritiansen (éd.), Settlement and Economy in Later Scandinavian Prehistory, British Archaeological Reports International, Series 211 : 43-62.

Malrain, F., Matterne, V., Méniel, P. 2002. Les paysans gaulois. Paris, Errance, 2002, 236 p.

MARChAND, C. 1997. « Réseau viaire et dessin parcellaire : étude morphologique de la région du Gâtinais oriental. » In Chouquer (dir.) 1997, Les Formes du paysage, Tome 3 : L'analyse des systèmes spatiaux, Paris, Editions Errance, 66-77.

Marchand, C. 2000. «Recherches sur les réseaux de formes. Processus dynamiques des paysages du sénonais occidental », thèse de doctorat, Université de Tours. 2 vol.

Marchand, C. 2003. «Des centuriations plus belles que jamais ? Proposition d'un modèle dynamique d'organisation des formes », Études Rurales, juillet décembre 2003, n 167-168 : 93-114.

Mathis, Ph. (dir.) 2003. Graphes et réseaux, modélisation multiniveau. Paris, Lavoisier, 361 p.

Mazoyer, M. et Roudart, L. 1997, 2002 (rééd). Histoire des agricultures du monde : du néolithique à la crise contemporaine, Paris, Seuil, $705 \mathrm{p}$.

Mennessier-Jouannet, C. et Buchsenschutz, O. 1996. " Des " champs celtiques » aux systèmes de parcellaire protohistoriques », in : CHOUQuer G. (dir.) 1996. Les Formes du paysage, Tome I : études sur les parcellaires : pré-actes du colloque d'Orléans (mars 1996). Paris, éditions Errance : 174-180. 
Ministère de l'Environnement et du Cadre de Vie 1980. Lutte contre les inondations - Méthode sommaire d'évaluation des critères économiques. Direction de la Prévention des Pollutions - Service de l'Eau - Sous-Direction des Eaux Continentales, 75 p. annexes

Mordant, C., Saligny, L. Poulet-Crovisier, N. « La circulation des objets métalliques à l'âge du Bronze entre Rhône et Saône: utilisation d'une transformation cartographique linéaire », Mappemonde $n^{\circ} 83$ (3-2006), en ligne sur http://mappemonde.mgm.fr.

Muraco, W.A. 1972. «Intraurban accessibility ». Economic Geography, 48 : 388-405.

Nordman, D. 1990. « La connaissance géographique de l'État (XIV $-\mathrm{e}$ XII ${ }^{\mathrm{e}}$ siècles) », in L'État moderne : le droit, l'espace et les forme de l'État, Paris, CNRS, 199 : 175-188.

Nuninger, L. et Sanders, L. 2006. «La modélisation des réseaux d'habitat en archéologie: trois expériences », Mappemonde n ${ }^{\circ} 83$ (3-2006), en ligne sur http://mappemonde.mgm.fr.

Ouzoulias, P. 1991. «Eudes Rigaud et le vieux chemin Paris-Rouen », in : Cuisenier J. (dir.), 1991. Matière et figure. Paris, La Documentation française, Collection études et Travaux n³ : 17-42.

Ozouf-Marignier M.-V. et Verdier N. 2000. "L'événement: un objet historique à emprunter", L'Espace Géographique, n³, 218-223.

Parish, Y.I.H., Müller, P. 2001. «Procedural Modeling of Cities », in : Proceedings of ACM SIGGRAPH 2001, ACM Press / ACM SIGGRAPH, New York. E. Fiume (ed), Computer Graphics, Annual Conference Series, ACM : 301-308.

Pelletier, M. 2002. Les cartes des Cassini, La science au service de l'État et des régions, Paris, CTHS. 338 p.

Pérol, C. 2004. « Cheminement médiéval, l'homme l'historien et la route », in Fray, J.L. et Pérol, C. L'historien en quête d'espaces, Clermont-Ferrrand, Presses Universitaires Blaise Pascal : 91-107.

Petitfrère C. 2003. « Dessine-moi un village... Le « village » sur la carte et le plan en Limousin sous l'Ancien Régime », in Tricard Jean (dir.), Le Village des Limousins : Études sur l'habitat et la société rurale du Moyen Âge à nos jours, Limoges, Presses universitaires de Limoges. 257-272.

Picon, A. 1992. L'invention de l'ingénieur moderne : l'École des ponts et chaussées, 1747-1851, Paris, Presses de l'École des ponts et chaussées. 767 p.

Picon, A. et Yvon, M. 1989. L'ingénieur artiste, Dessins anciens de l'École des ponts et chaussées, Paris, Presses de l'École des ponts et chaussées. 206 p.

Pinchemel P. 1944. « Habitat Rural et Maisons Rurales en Picardie », Bulletin de l'association des géographes français, $\mathrm{n}^{\circ}$ 163-166. 102-108.

Pinchemel, P. et Pinchemel, G., 1988. La face de la terre, éléments de géographie. Paris, Armand 
Colin, $515 \mathrm{p}$.

Pion, P. 1990. « De la chefferie à l'État? Territoires et organisation sociale dans la vallée de l'Aisne aux Âges des métaux (2200-20 av. J-C.) », Archéologie et Espaces, Actes des Rencontres Internationales d'Archéologie et d'Histoire d'Antibes (10 ; 1989), Editions APDCA, Antibes : 183260.

PIтTs, F. 1965. « A graph theoretic approach to historical geography ». The Professional Geographer, $\mathrm{n}^{\circ} 17: 15-20$.

Prou, M. 1884. Les coutumes de Lorris, Paris, 176 p.

Revel, J. 1992. «La région », in, Les lieux de mémoire, III, Les France, 1, Conflits et partages, nrf, Gallimard : 850-883.

Robert, S. (à paraître). «Des outils pour l'analyse de la transmission des trames et réseaux planimétriques : approches multiscalaires et traitements sur SIG», in: direct Colloque du réseau thématique pluridisciplinaire "Paysage et environnement, de la reconstitution du passé aux modèles prospectifs », Chilhac 27-30 sept. 2006.

Robert, S. 1996. «Le Parcellaire du plateau de Sénart (Seine-et-Marne)», in: Chouquer G. (dir.) 1996 : Les Formes du paysage, Tome I : études sur les parcellaires : pré-actes du colloque d'Orléans (mars 1996). Paris, éditions Errance : 11-27.

Roвert, S. 1997. «Le relevé parcellaire sur photographies aériennes et cartes anciennes », in : Chouquer G. (dir.) 1997. Les Formes du paysage, Tome 3 : L'analyse des systèmes spatiaux, Paris, Editions Errance : 88-95

Robert, S. 2004. L'analyse morphologique des paysages entre archéologie, urbanisme et aménagement du territoire. Exemples d'études de formes urbaines et rurales dans le val d'Oise, thèse de doctorat, Université de Paris 1, 2 vol.

Roвert, S. 2006a. «La résilience des réseaux routiers : l'exemple du Val-d'Oise », in : Bulletin AGER $n^{\circ} 15$, année 2005, Besançon, Presses Universitaires de Franche-Comté, 2006 : 8-14.

RoBert, S. 2006b. «Les itinéraires anciens traversant le département du Val-d'Oise », in : Bulletin archéologique du Vexin Français et du Val-d'Oise, n³8 : 7-23

Robert, S. 2007. « Modeling Ancient Roads », in : Medieval Europe Paris 2007, 4e Congrès international d'Archéologie Médiévale, Institut National d'Histoire de 1'Art, Paris, 3-8 septembre 2007. en ligne sur http://medieval-europe-paris-2007.univ-paris1.fr.

Roncayolo, M. 1988. "la morphologie, entre la matière et le social", Villes en parallèle, n¹2-13, $42-$ 59.

Rouche, M. 1982. «L'héritage de la voirie antique dans la Gaule du haut Moyen-Age (Ve-XIe 
siècle) », in : Flaran, 1982. «L'homme et la route en Europe occidentale au Moyen Age et aux Temps Modernes », in : Cahier de Flaran, $n^{\circ} 2$, Auch. : 12-32.

SAHLINs, P. 1996. Frontières et identités nationales, la France et l'Espagne dans les Pyrénées depuis le XVIIe siècle, Paris, Belin ( $1^{\mathrm{er}}$ ed. en anglais 1989), $416 \mathrm{p}$.

Sakarovitch, M. 1984. Optimisation combinatoire. Méthodes mathématiques et algorithmiques. Programmation discrète, Paris, Hermann, 269 p.

SAn JuAn, G. 2005. « La Normandie dans l'âge du Bronze européen », in: C. Marcigny, C. Colonna, E. GHesquière et G. Verron (dir.), La Normandie à l'aube de l'histoire, Les découvertes archéologiques de l'âge du Bronze 2300-800 av. J.-C.. Paris, Somogy : 17.

Schnapp A. 1993. La conquête du passé. Aux origines de l'archéologie. Paris, éditions Carré, 511 p.

Shefri, Y. 1985. Urban Transportation Networks - Equilibrium Analysis with Mathematical Programming Methods. Prentice Hall, 400 p.

Spratt, D.A. 1981. "Prehistoric boundaries on the North Yorkshire Moors », in: G. Barker (éd), Prehistoric communities in Northern England : Essays in social and Economic reconstruction, Sheffield, University of Sheffield Press : 87-104.

TissieR, J.-L. 2003. « Chemins et géographie », Les carnets du paysage, $\mathrm{n}^{\circ} 11: 35-53$

Touzery M. 1995. Atlas de la généralité de Paris au XVIII siècle : un paysage retrouvé, Paris, Comité pour l'histoire économique et financière de la France. 176 p.

Van Leusen, P. M. 2002. Pattern to process : methodological investigations into the formation and interpretation of spatial patterns in archaeological landscapes. Groningen, Rijkuniversiteit. 365 p.

Verdier N. 2002. « Poste et territoires : évolution de la pensée du territoire chez les administrateurs de la Poste au XIXème siècle ", in : Le Roux (dir), Histoire de la poste. De l'administration à l'entreprise, Editions rue d'Ulm : 61-86.

Verdier N. 2004. " L'échelle dans quelques sciences sociales : petite histoire d'une absence d'interdisciplinarité ", in O. Orain, D. Pumain, C. Rozemblat et N. Verdier, Géographie, échelles et temporalités en géographie, Paris, CNED : 25-56.

Verdier N. 2007. «Le réseau technique est-il un impensé du XVIIIe siècle : le cas de la poste aux chevaux », Flux, Cahiers scientifiques internationaux Réseaux et Territoires, 68 : 7-21.

VERDIER N. 2008a. «Gerarchie urbane e città in rete : l'urbanizzazione della Francia tra 1700 e 1830 », in Iachello E. e Militello P. (dir.), L'insediamento nella Sicillia d'étà moderna et contemporanea, Bari, Epuglia.

Verdier N. 2008b. "Modeler le territoire : les ingénieurs des Ponts et chaussées et leurs usages de la 
carte (fin XVIIe-début XIXe siècles)", in Isabelle Laboulais (dir.), Les usages des cartes (XVIIe-XIXe siècles, pour une approche pragmatique des productions cartographiques, Strasbourg, Presses universitaires de Strasbourg, 2008, 51-66 et VI-VII.

Verhagen P., Gili S., Mico R., Risch R. 1999. « Modelling Prehistoric Land Use Distribution in the Rio Aguas Valley (SE Spain) », in: Dingwall L. et al. (eds) : Archaeology in the Age of the Internet. Proceedings of the CAA97 conference, Oxford, BAR International Series, 750, CDRom.

Vidal De Lablache, P., 1902. "Routes et chemins de l'ancienne France », Bulletin de géographie historique et descriptive, $\mathrm{n}^{\circ} 17: 115-126$.

Vidal, L., Pomarèdes, H. et Sauvage, L. 1996. «Traces matérielles de la mise en valeur et de l'aménagement des campagnes aux portes de Nîmes : les parcellaires antiques de la plaine du Vistre », in : Chouquer G. (dir.) : Les Formes du paysage, Tome II : études sur les parcellaires : actes du colloque d'Orléans (mars 1996). Paris, éditions Errance, 1996 : 57-66.

Vignon E. 1862. Études sur l'administration des voies publiques en France aux dix-septième et dixhuitième siècles, Paris, Dunod. 3 vol. XIII-146-145, 213-358, 135-281 p.

Vion, E. 1989. «L'analyse archéologique des réseaux routier : une rupture méthodologique, des réponses nouvelles », Paysages découverts $I$ : 67-69.

Vita-Finzi, C. et Higgs, E.S. 1970. « Prehistoric economy in the Mount Carmel area of Palestine: site catchment analysis », Proceedings of the Prehistoric Society, 36 : 1-37.

Voyages et Voyageurs au Moyen Age, 1996. Société des historiens médiévistes de l'enseignement supérieur public. Voyages et Voyageurs au Moyen Age. XXVIe Congrès de la SHMES (LimogesAubazine, mai 1995), Paris, Publications de la Sorbonne, 314 p.

Wabont, M., Abert, F., Vermeersch, D. 2006. Le Val-d'Oise - 95. In : Provost M. (dir.), Carte Archéologique de la Gaule. Le département du Val-d'Oise. Paris : Editions Les Belles Lettres, 495 p.

Wasserman, S., Faust, K. 1994. Social Network Analysis - Methods and applications. Cambridge University Press, 857 p.

Watteaux, M. 2004. "Le plan radio-quadrillé des terroirs non planifiés », in : Chouquer G. (prés.). Etudes Rurales $n^{\circ} 167-168.2004: 187-214$.

Weber, J. 2000. «Using GIS to Model and Visualize Congestion Effects on Individual Accessibility », in : Proceedings of the Annual Meeting of the East Lakes and West Lakes Divisions of the Association of American Geographers, Oxford, Ohio, $18 \mathrm{p}$.

Zaksek, K., Fovet, E., Nuninger, L. et Podobnikar T. 2007. « Path modelling and settlement pattern », in: Computer Applications and Quantitative methods in Archaeology, Berlin, , Germany, April 2-6, 2007. Bonn (Germany): Habelt (Kolloquien zur Vor- und Frühgeschichte, vol. 10. 
